\title{
Two-dimensional impedance imaging of cell migration and epithelial stratification $\dagger$
}

\author{
Pontus Linderholm, ${ }^{* a}$ Thomas Braschler, ${ }^{a}$ Jeanne Vannod, ${ }^{b}$ Yann Barrandon, ${ }^{b}$ Michel Brouard ${ }^{b}$ and \\ Philippe Renaud ${ }^{a}$
}

Received 15th March 2006, Accepted 29th June 2006

First published as an Advance Article on the web 3rd August 2006

DOI: $10.1039 / \mathrm{b} 603856 \mathrm{e}$

We present a miniaturized impedance imaging system, developed for $2 \mathrm{D}$ imaging of cell and tissue culture. The system is based on 16 microelectrodes $(5 \mu \mathrm{m} \times 4 \mathrm{~mm})$. An equivalent circuit for fourpoint (tetrapolar) impedance spectra was developed and validated. The system uses an Agilent 4294A impedance analyser combined with a front-end amplifier for the impedance measurements. Human epithelial stem cells (YF 29) were grown on the device surface. Cell migration speeds of $300 \mathrm{~nm} \mathrm{~min}{ }^{-1}$ following a "scratch" wound closure assay could be established. Using a commercial software developed for geophysical prospecting, we could generate impedance tomography images at $10 \mathrm{kHz}$ revealing cell migration, increase of epithelial thickness and changes in tissue resistivity over a time course of several days.

\section{Introduction}

Electrical characterization of cell cultures has been used for more than a century to study the permeability and transportation properties of epithelia, such as epidermis in the skin. ${ }^{1-3}$ Typically, cells are cultured on a filter with electrodes on both sides that measure the transepithelial resistance (TER). Such a system gives no spatial information regarding the resistivities. We report here on a novel technique which extends the traditional TER measurements to actual 2D imaging of vertical sections of the tissue culture. The technique can be used to study proliferation, differentiation and migration of three-dimensional tissue culture.

The presented technique is a miniaturization of a technique commonly used in geophysical surveys, called "resistivity imaging". Resistivity imaging is based on low frequency impedance measurements from electrodes placed on the ground surface or in boreholes, and has been used to study subsurface structures and phenomena such as permafrost, ${ }^{4}$ groundwater flow ${ }^{5}$ and metal ores. ${ }^{6}$ In the biomedical sciences, a technique based on the same principles has been developed under the name electric impedance tomography (EIT). ${ }^{7}$ Here the electrodes are arranged in a circle around the sample, typically the torso or the head. Research applications of EIT include pulmonary ventilation, ${ }^{8}$ blood flow, ${ }^{9}$ and brain activity. ${ }^{10}$

As suggested by Brown et al. in 1985, it should be possible to obtain images with a resolution on the order of a few microns by using sufficiently small electrodes. ${ }^{11}$ Recently, there have indeed been reports on miniaturized EIT systems, ${ }^{12-14}$ but the

${ }^{a}$ Laboratory of Microsystems, Swiss Federal Institute of Technology, 1015, Lausanne, Switzerland. E-mail: pontus.linderholm@epfl.ch; Fax: +4121693 59 50; Tel: 0041216936575

${ }^{b}$ Laboratory of Stem Cell Dynamics, Swiss Federal Institute of Technology, 1015, Lausanne, Switzerland

$\uparrow$ Electronic supplementary information (ESI) available: Video of migration in the cell layer. See DOI: 10.1039/b603856e smallest object presented so far is a $80 \mu \mathrm{m}$ metal wire. Given that the subject has been under investigation for several decades, it is somewhat surprising that there have not been more reports on miniaturized impedance imaging systems. In this article, we will show that the use of microelectrodes places particular constraints on the instrumentation and the frequencies used. Previous work on impedance modeling has often neglected the electrode impedances and the stray capacitances between the metal leads, ${ }^{15}$ or the capacitive pathways between the voltage pick-up and ground. ${ }^{16}$ For these reasons, we developed an electrical model which can accurately model the frequency response of four-point measurements using microelectrodes. The model is based on analytical expressions for the resistances between the electrodes and can as such be useful for electrode optimizations in other fourpoint applications as well.

Compared to other imaging modalities, tomography based on electric impedance presents several attractive features such as relatively cheap and harmless equipment, as well as the absence of invasive measurements and movable parts. Apart from its obvious applications in wound healing studies and TER investigations, potential biomedical applications of a microimpedance tomography system include in vitro monitoring of cell electroporation, ${ }^{17}$ wound healing characterization and cell migration. ${ }^{18}$

Wound healing assays, where the cells are removed from an area using a scalpel or the tip of a pipette, are a standard tool in cell migration studies. We report here for the first time the use of impedance imaging to monitor cell cultures, in particular the creation of cross-sectional images of cells migrating across a mechanically induced wound. Using an array of 16 electrodes, we show that it is possible to follow cell migration and tissue thickness simultaneously. We present results validating the basic functionality of the device, as well as reconstructed resistivity side-views of pluristratified epithelia using human keratinocyte stem cells. We give both experimental and theoretical data showing that the bandwidth 


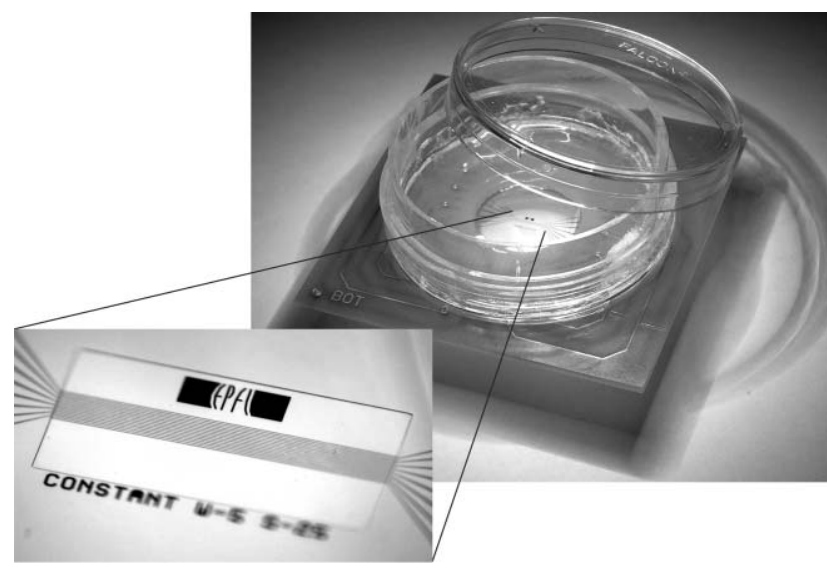

Fig. 1 Photograph of the device with the cell culture chamber. The electrode array consisted of 16 linear electrodes, $5 \mu \mathrm{m}$ wide and $4 \mathrm{~mm}$ long, patterned on a glass substrate mounted on a PCB. The cells grow over the entire chip surface.

of four-point measurements depends highly on stray capacitances and electrode dimensions.

\section{Theory}

\section{Four-point measurements}

Impedance tomography makes use of four-point (tetrapolar) measurements, i.e. the current injection electrodes are separated from the voltage pick-up electrodes. The reason is that the four-point measurements are less sensitive to interface impedances and electrode polarization, and have a greater penetration depth.

Using an array of electrodes (Fig. 1), a large number of different measurement configurations can be made. However, some of these configurations will result in very poor data quality. For instance, the voltage between two neighboring pickup electrodes will be very small if they are not placed between the current injection electrodes. A plethora of so-called "measurement arrays", i.e. sets of different measurement configurations, have been devised with the aim of reducing the number of measurements needed to recreate an image, while keeping an acceptable spatial resolution and signal-to-noise ratio. ${ }^{19}$ We used the so-called WennerSchlumberger (W-S) arrangement, in which the injection electrodes are symmetrically situated around the pick-up electrodes (Fig. 2a).

The number of different measurements using the $\mathrm{W}-\mathrm{S}$ arrangement is:

$$
n=\sum_{i=1}^{N-3} \sum_{j=i+3}^{N}\left\lfloor\frac{j-i-1}{2}\right\rfloor
$$

where $N$ is the number of electrodes and the $\lfloor$ and $\rfloor$ symbols denote the floor function. For 16 electrodes this gives 252 measurements.

Fig. 2(a) gives a schematic representation of the equipotentials for a homogeneous medium in the basic tetrapolar measurement geometry employed. The voltage pick-up electrodes measure the potential between the two equipotential lines associated with each electrode. The bulk resistance is then the resistance measured between these two lines.

Regions in close proximity to the electrodes have the greatest influence on the measured signal, but the contribution of the different depths to the total signal depends on the electrode geometries. The median depth of investigation, i.e. the depth below which half of the total power is dissipated, is a common means of characterizing the probe depth. ${ }^{19,20}$ For linear electrodes the median depth of investigation is:

$$
z_{\text {median }}=\frac{1}{2} \sqrt{\left|a^{2}-b^{2}\right|}
$$

where $a$ is half the distance between the current injection electrodes, and $b$ is half the distance between the pick-up electrodes. The probe depth thus increases as the pickup electrodes are brought closer together or when the spacing between the injection electrode increases with the pick-up electrodes at fixed positions. The typical thickness of the epithelial culture that we are interested in is $10-100 \mu \mathrm{m}$. Choosing a center-center spacing of $30 \mu \mathrm{m}$ between the electrodes, we obtain median probe depths ranging from $21 \mu \mathrm{m}$ a)

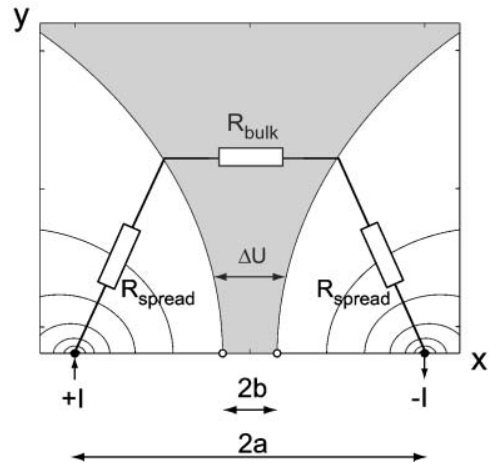

b)

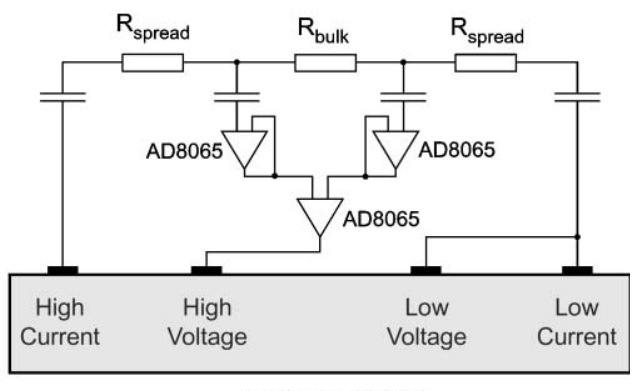

Agilent 4294A

Fig. 2 (a) A schematic of the tetrapolar measurement configuration. As the distance between the pick-up electrodes decreases, the measured impedance will decrease since the potential difference decreases, but the measurement will also become more sensitive to changes deep inside the sample. (b) A custom instrumentation amplifier was used to buffer and then subtract the potentials at the two pickup electrodes. The difference was fed into the high potential port of the Agilent 4294A, the low potential port was connected to the virtual ground provided by the current measurement port. 
(13 measurements) to $112 \mu \mathrm{m}$ (1 measurement) for the 252 different measurement configurations.

\section{Apparent resistivity}

For a homogeneous sample, the resistance is proportional to the resistivity:

$$
R=\kappa \rho
$$

where $\kappa$ is a geometrical factor called the cell constant, and $\rho$ is the resistivity of the sample. This general expression is valid for both tetrapolar and bipolar (two-point) measurements, but with different expressions for the cell constant.

For layered structures, the measured resistance will depend on the layer thicknesses and their resistivities in a non-linear way. In order to compare measurements from different electrode geometries, the resistance values are therefore normalized with the cell constant of the corresponding geometries. The resulting value can be thought of as the resistivity of an equivalent homogeneous sample yielding the same resistance as the heterogeneous sample. This resistivity is called the apparent resistivity:

$$
\rho_{\text {apparent }}=\frac{R}{\kappa}
$$

If the sample is homogeneous, all electrode geometries will measure the same apparent resistivity. However, if the top layer is more resistive than the bottom layers, the apparent resistivity for measurement arrangements having a large penetration depth will be higher than those arrangements with a small penetration depth.

\section{Tetrapolar impedance spectroscopy}

Four-point measurements are used to eliminate the impedances of cables and contacts. This has been used to "bypass" the interfacial impedance of the electrodes in order to make measurements at low frequencies. ${ }^{21}$ However, as we shall explain here, the use of microelectrodes significantly reduces the bandwidth of the tetrapolar measurements.

The perfect tetrapolar impedance measurement of a purely resistive sample is independent of the applied frequency, as long as stray capacitances are ignored. However, at low frequencies the electrode impedance will surpass that of the voltmeter, and one would expect the measured voltage to decrease and the impedance to fall to zero. In this "classical" view of the four-point spectrum, the impedance therefore starts near zero, increases to the bulk resistance value and then drops again. ${ }^{22}$

Consequently, we were a bit surprised when we started recording the tetrapolar spectra that there was sometimes a "bump" in the impedance magnitude before it decreased at high frequencies due to the parasitic capacitances. An equally puzzling effect was that the impedance also increased from the plateau value as the frequency decreased.

It turns out that these anomalies can be attributed to the separation of the current and voltage measurements in combination with very high electrode impedances. Stray capacitances between the leads will tend to act as voltage

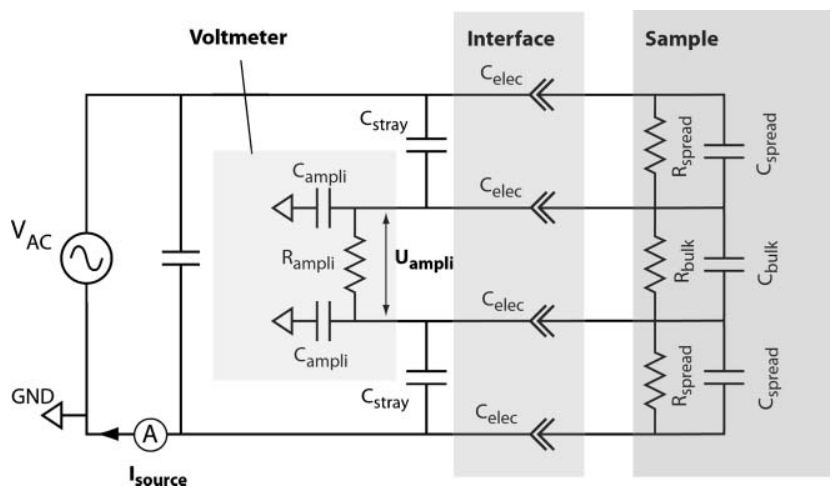

Fig. 3 Equivalent circuit for the tetrapolar measurement. The electrode impedances and stray capacitances were determined through calibration measurements, whereas all of the sample-dependent elements were modelled analytically.

dividers and have a considerable effect on the impedance spectra. A second consequence of the separation of current and voltage measurements is that the portion of current passing through the voltmeter to ground influences the measured voltage, but is not necessarily measured by the current measurement. We propose an equivalent circuit for analyzing the four-point spectra, which takes into account both these effects (Fig. 3).

The impedance of the sample can be modelled analytically. First, using the definition of the cell constant (eqn (3)), the bulk resistance is given by:

$$
R_{\text {bulk }}=\kappa_{4 \mathrm{pt}} \rho
$$

where the tetrapolar cell constant $\left(\kappa_{4 \mathrm{pt}}\right)$ for electrodes symmetrically positioned around the origin is given by: ${ }^{23}$

$$
\kappa_{4 \mathrm{pt}}=\frac{R}{\rho}=\frac{\Delta V \sigma}{I}=\frac{1}{L \pi} \ln \left(\frac{(a+b)^{2}}{(a-b)^{2}}\right)
$$

where $L$ is the length of the electrodes, $a$ is half the distance between the current injection electrodes, and $b$ is half the distance between the voltage pick-up electrodes. This approximation assumes that the electrodes are linear and neglects fringing fields at the end points of the electrodes.

The spreading resistance (see Fig. 2) is then given by

$$
R_{\text {spread }}=\frac{\kappa_{\text {bipolar }} \rho-R_{\text {bulk }}}{2}
$$

The cell constant of a bipolar measurement is given by ${ }^{24,25}$

$$
\kappa_{\text {bipolar }}=\frac{2}{L} \frac{K\left(\frac{s^{2}}{(s+2 w)^{2}}\right)}{K\left(1-\frac{s^{2}}{(s+2 w)^{2}}\right)}
$$

where $L$ is the in-plane length of the electrodes, $s$ is the distance between the electrodes, $w$ is the width of the electrodes and $K$ is the elliptic integral of the first kind. The elliptic integral in this case is a function of the electrode width and gap. 
In an aqueous solution, there is also a capacitive pathway for the current, which can be modelled as a capacitor in parallel with the resistance of the sample. The bulk capacitance is given by:

$$
C_{\text {bulk }}=\frac{\varepsilon_{0} \varepsilon_{\mathrm{r}}}{\kappa_{4 \mathrm{pt}}}
$$

where $\varepsilon_{0}$ is the permittivity of vacuum and $\varepsilon_{\mathrm{r}}$ is the relative permittivity of water $(\sim 78)$. In analogy with the expression for the spreading resistance we find:

$$
C_{\text {spread }}=\frac{2 \varepsilon_{0} \varepsilon_{\mathrm{r}}}{\kappa_{\text {bipolar }}-\kappa_{4 \mathrm{pt}}}
$$

Accurate models for the electric interface are often complicated to the degree of being practically useless. We have therefore resorted to a more empirical model to describe the interface impedances of the electrodes, i.e. a constant phase element:

$$
Z_{\text {electrode }}=\frac{1}{T(j \omega)^{\alpha}}
$$

where $T$ and $\alpha$ are experimentally determined parameters. These two parameters should be determined through fitting to bipolar impedance spectra. Note that the stray capacitances on the circuit board and the electrode impedances are the only experimentally determined values in the equivalent circuit in Fig. 3. Once these are determined through calibration measurements, the only free parameter that remains is the resistivity of the solution, assuming that $\varepsilon_{\mathrm{r}}$ is a constant in the frequency range of interest to us.

\section{Materials and methods}

\section{Device fabrication}

Ti-Pt (20 nm-100 nm) microelectrodes were patterned on floatglass using standard lift-off procedures. The microelectrodes were rectangular, $5 \mu \mathrm{m}$ wide, $4 \mathrm{~mm}$ long and separated by a gap of $25 \mu \mathrm{m}$ (Fig. 1). A $10 \mu \mathrm{m}$ layer of polyimide (PI 2731) was used to isolate the leads from the electrodes to the pads. After dicing the chips were bonded to a PCB, and a culture chamber (id $35 \mathrm{~mm}$ ) was mounted on top of the array. The cell constants of all 252 injection schemes were determined by linear fitting of the measurements in $\mathrm{NaCl}$ solutions.

\section{Cell culture}

Frozen stocks of human epidermal keratinocytes YF29 isolated from the foreskin of a newborn were thawed and cultured between passages 3 and 8..$^{23,24}$ They were cultured on a feeder layer of lethally irradiated mouse embryonic 3T3 fibroblasts. The cultures were kept in a $10 \% \mathrm{CO}_{2}$ atmosphere at $37^{\circ} \mathrm{C}$. The medium, cFAD (a $3: 1$ mixture of the DulbeccoVogt modification of Eagle's medium and Ham's F12 medium; Gibco BRL) supplemented with 10\% fetal calf serum (Hyclone Laboratories Inc.) and $10 \mathrm{ng} \mathrm{ml}^{-1}$ human recombinant EGF as described previously, ${ }^{23,24}$ was changed every 2 days. Reference cultures were cultured on commercial polystyrene culture dishes (Falcon 35-3001, Becton-Dickinson France SA). The measurements began when the cells had reached confluence.
An inverted microscope (Axiovert 200 M, Carl Zeiss AG) equipped with differential interference contrast (DIC) filters was used for the reference measurements of the cell culture thickness. The microscope was equipped with an XL Incubator and a $\mathrm{KH} \mathrm{CO}$-Cover (Carl Zeiss AG), under which the cells were placed during measurements. The focal depth of the microscope could be adjusted with a precision of $100 \mathrm{~nm}$, and the thickness was measured by focusing on the electrode edges, and then on the top of the cellular layer. Time lapse imaging was performed using the commercial software AxioVision (Carl Zeiss GmBH).

\section{Impedance acquisition}

The impedance was measured from $100 \mathrm{~Hz}$ to $1 \mathrm{MHz}$ using an Agilent 4294A impedance analyser, modified with a front-end amplifier to allow for true four-point measurements ${ }^{26}$ (Fig. 2b). The impedance analyzer was controlled by a proprietary Java application via a USB-GPIB interface. The RMS voltage amplitude was $100 \mathrm{mV}$. An analog crosspoint switch array (AD75019), controlled via the parallel port of the PC, was used to connect the electrodes to impedance analyser. The time resolution depends on the number of measurements and the acceptable noise-level, in this study a set of measurements was usually performed in 10 seconds.

\section{Tomographical reconstruction}

Creating a tomographical image from the normalized impedance measurements consists of finding the resistivity distribution that best explains measured data. Calculating the resistance for a given electrode geometry based on the internal resistivity distribution of a sample is referred to as solving the forward problem. Consequently, finding the resistivities based on resistance measurements is called solving the inverse problem. An excellent tutorial on least-squares inversion is given by Lines and Treitel. ${ }^{27}$ In this paper, we used the commercial software RES2DINV (GeoTomo Software, Penang, Malaysia) to inverse the apparent resistivities to find the resistivity of the sample.

The program was developed for geophysical surveying where the electrodes can be considered as point-like. Since the electrodes in this study are linear, the reconstructed images will be distorted, where the degree of distortion will increase as the vertical position increases.

The program uses a smoothness-constrained least-squares method which penalizes rapid changes in resistivity in the sample in order to find the best distribution of resistivities. The optimization method basically tries to minimize the difference between the calculated and measured apparent resistivities by adjusting the resistivities of the internal elements of the structure. A measure of the accuracy of the reconstructed images is usually reported as the root-mean-squared error:

$$
\mathrm{RMS}=\sqrt{\frac{\left\|\left(\boldsymbol{m}(\boldsymbol{\rho})-\boldsymbol{m}^{*}\right)\right\|^{2}}{N_{\text {elem }}}}
$$

where $\boldsymbol{\rho}$ is the resistivity vector, $\boldsymbol{m}(\boldsymbol{\rho})$ is the solution to the forward problem using the resistivity distribution $\boldsymbol{\rho}, \boldsymbol{m}^{*}$ is the vector with the observed apparent resistivities, and $N_{\text {elem }}$ is the number of elements in the model. 


\section{Results and discussion}

\section{Four-point spectra}

We expanded the model for four-point spectra proposed by $\mathrm{Lu}$ et $a l^{16}$ by introducing two more stray capacitors, separating the spreading resistance and the electrode impedance in two, and defining a shunting capacitance from the pick-up leads to ground. Furthermore, we used analytical expressions for the calculation of all sample-dependent parameters, yielding a theoretical expression where the only free parameter was the sample resistivity. A large range of solution resistivities can thus be rapidly analyzed for arbitrary electrode geometries without resorting to finite element simulations to find the sample resistances.

At low frequencies, the interfacial impedance at the microelectrodes becomes very high compared to $R_{\text {spread }}$ and $R_{\text {bulk }}$, which essentially means that the entire sample will be at half the potential of the current source (providing that the two current electrodes are of equal size). As long as the input impedance of the instrumentation amplifier is very high, the pick-up electrode and the stray capacitance between the injection and pickup leads will form a voltage divider. This has the regrettable result that the measured voltage depends neither on sample resistivity nor frequency. The instrumentation amplifier will then measure a frequency-independent potential, given by the ratio of stray capacitance between current injection and pickup electrodes, and the interfacial capacitance of the pickup electrodes. The current, however, still depends on the frequency, and the impedance therefore increases as the frequency decreases. This increase in impedance must therefore not be confused with a so-called $\alpha$-dispersion.

The high-frequency bump $(\sim 1 \mathrm{MHz})$, is due to the capacitive coupling between the amplifier inputs and ground. This current is effectively not seen by the impedance analyser, since it returns via ground and not the measurement cables (note the position of ground in the equivalent circuit in Fig. 3). Taken together, these two effects limit the frequency range readily available for tetrapolar impedance measurement, where the limits depend on the electrode sizes and the resistances measured.

The tetrapolar impedance spectra corresponded nicely to the theoretical predictions (Fig. 4). The capacitance between the voltmeter leads and the injection leads was $1 \mathrm{pF}$, the capacitance between the voltmeter input and ground $20 \mathrm{pF}$, and the stray capacitance between the two injection lines was $1 \mathrm{pF}$.

Accurate tetrapolar measurements are thus only possible within a limited frequency range around $10 \mathrm{kHz}$. Care must therefore be taken in the design of the electronics, which partially explains why tetrapolar measurements using microelectrodes are still quite uncommon in lab-on-a-chip applications.

\section{Apparent resistivity}

The cell constants of all the measurement schemes were determined to verify the correct functioning of the electrodes and to yield accurate apparent resistivities for the reconstruction algorithm.
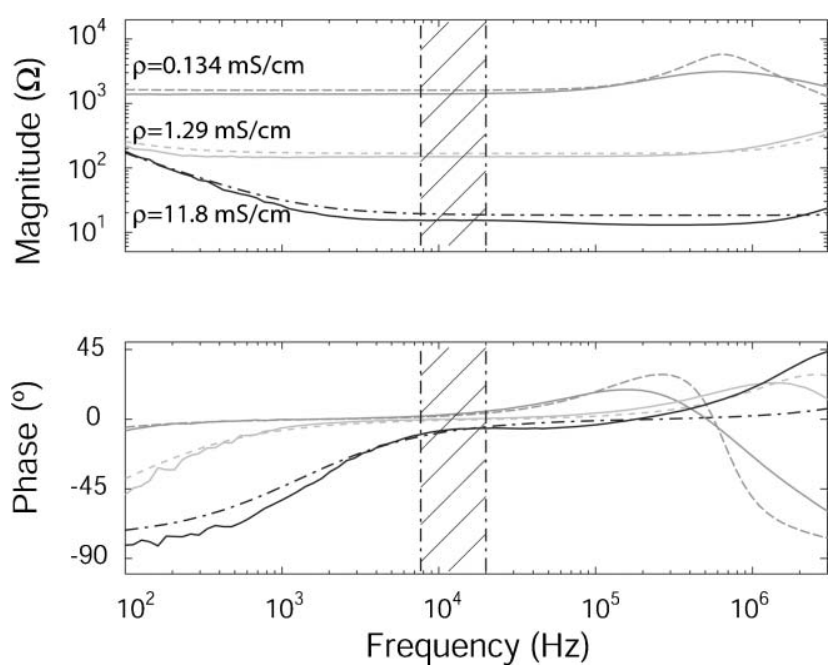

Fig. 4 Bode plots of the tetrapolar measurements in $\mathrm{KCl}$ solutions of different conductivities (solid lines) and the fits based on the equivalent model (dotted lines). The only free variable is the resistivity $\rho$.

The four-point cell constants decrease as the spacing between the injection electrodes is increased, if the distance between the pick-up electrodes is kept constant (Fig. 5a). The difference between the measured cell constants and the theoretical predictions (eqn (6)) was less than $10 \%$. The measurements were carried out at $10 \mathrm{kHz}$. The slight discrepancy is attributed to the line-approximation of the rectangular electrodes. The electrode interfacial impedances were determined by fitting to the bipolar spectra (Fig. 5b). The $T$ parameter of the CPE (eqn (11)) was found to be $6.7 \times 10^{-9}$ and the $\alpha$ parameter 0.93 .

\section{Cell culture}

The impedance monitoring of the cell culture began when the human keratinocytes covered the device surface with a confluent layer of cells (six days after seeding). The lethally irradiated mouse fibroblasts had by then been pushed away from the surface by the human cells and then been rinsed away during medium changes. After confluency, the keratinocytes started differentiating, forming a pluristratified epithelium, which resulted in a thickness increase.

At a frequency of $10 \mathrm{kHz}$ the cells can be considered isolating, due to the blocking properties of the cell membrane capacitances, if the culture medium has a physiological resistivity $(\sim 0.7 \Omega \mathrm{m}){ }^{28}$

To study migration, the cells on the electrodes were removed using a scalpel. Four cuts were made around the cells, and the rectangular cell layer was then gently removed using a pair of tweezers. On one side of the sensing surface, the cut was made close to the electrodes, whereas on the other side it was made far from the electrodes so that the migration would be from one direction only. Care was taken that the cuts were made parallel to the electrodes (Fig. 6a).

After about 10 hours, the cells started migrating back to the electrodes. As the cell border extended across a certain electrode, it could be seen how the impedance rose sharply (Fig. 6b). In consistency with eqn (2), the measurement 
a)

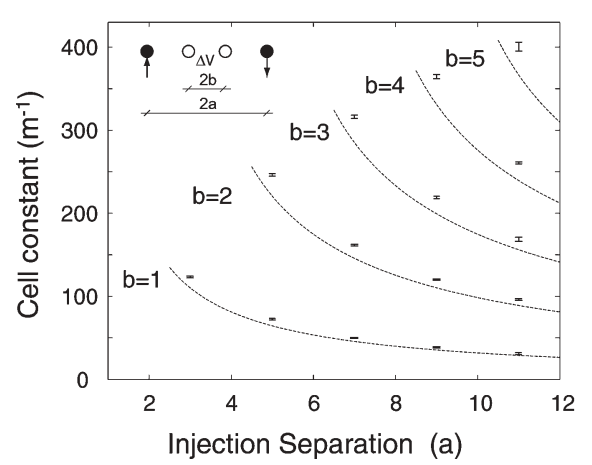

b)

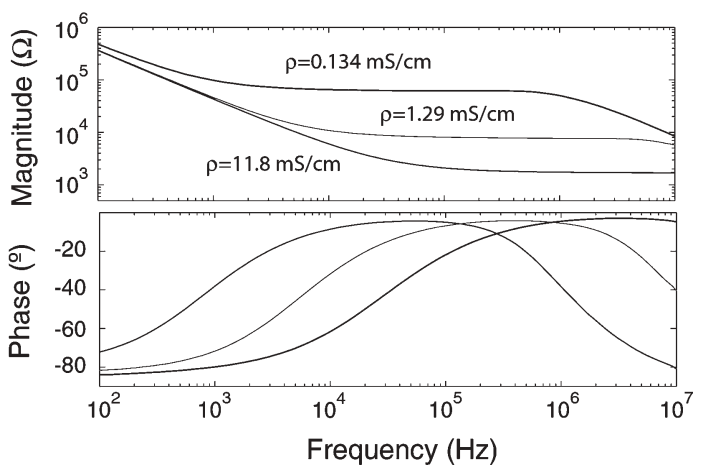

Fig. 5 (a) The theoretical four point cell constants (dotted lines) approximating the electrodes as 2D points worked well to model the experimentally determined cell constants. The error bars are to scale. The parameter $a$ is the distance between the origin and the injection electrodes, and the $b$ parameter is the distance from the origin to the pick-up electrode. (b) Bipolar spectra of the impedance between the 1st and the 16th electrodes. The low frequency part was used to extract parameter values for the electrode interface impedance.

a)

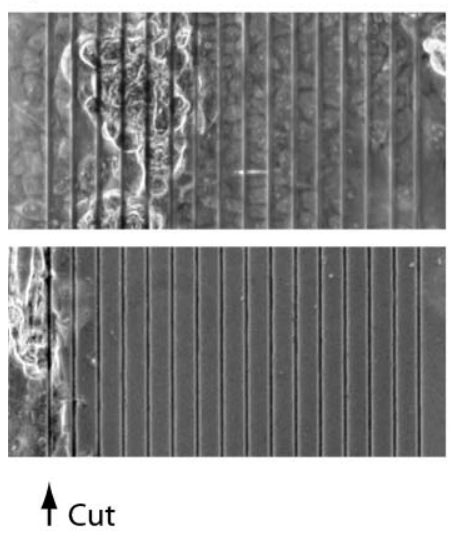

b)

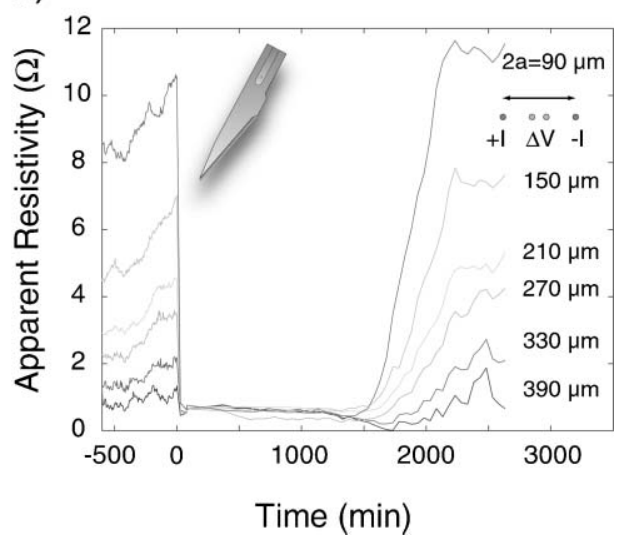

Fig. 6 (a) Micrographs of the cell culture growing on the device surface, where the cells on the electrodes have been removed mechanically. The thin vertical lines are the $5 \mu \mathrm{m}$ platinum electrodes. The agglomerations in the top image are differentiated cells. (b) The apparent resistivities for six electrode configurations having different probe depths.

configurations where the injection electrodes were close together were more sensitive to the cell layer than configurations where the injection electrodes were far apart (Fig. 6b).

The cell layer, even after differentiation had started, was in constant motion. There were concerted movements of cells over several millimetres, as well as tiny oscillatory motions by a few groups of cells (see supplementary video $\dagger$ ). This constant migration has been shown to be responsible for the fine structure of the impedance variation over time $e^{18}$ (Fig. 6b).

If the impedance increase is shown for measurements with identical electrode separations ( $a=45, b=15$ ), the movement of the cell frontier can be clearly seen (Fig. 7b).

Assuming that the cells first migrate across the surface before they start differentiating, a given apparent resistivity value should correspond to the same thickness for all electrode geometries with the same injection/pickup ratio. By comparing the time intervals at which the apparent resistivity had risen to $1 \Omega \mathrm{m}$, we could therefore calculate a migration speed of $340 \pm 20 \mathrm{~nm} \mathrm{~min}^{-1}$ (95\% confidence interval). This value was independent of the measurement scheme used. Furthermore, it was constant throughout the migration process (Fig. 7a).
Mouse epidermal stem cells have been shown to migrate $\sim 1 \mathrm{~mm}$ in 24 hours following a scratch test, which corresponds to a migration speed of approximately $700 \mathrm{~nm} \mathrm{~min}{ }^{-1}{ }^{29}$ Considering that we used multi-layered cultures, it seems reasonable that we would measure a somewhat lower migration speed.

The image reconstruction was carried out once the measurements were terminated. Each set of 252 apparent resistivities resulted in one image. It was found that using the built-in robust constraint on the inversion process resulted in the best image quality. The images were obtained using an initial damping factor of 0.3 . Due to the smoothness constraints, there is no clear boundary between the cells and the surrounding medium. However, without this constraint the inversion process yielded unacceptably high error functions.

Fig. 8 shows a sequence of images taken each 200 minutes. It can be clearly seen how the cells slowly crawl over the surface, and how they start increasing in thickness. The RMS error for these images varied between 13-32\% (20.3 \pm 5.7\%, 95\% confidence interval). These errors are quite large, but we believe they are mainly due to the point-electrode assumption of the reconstruction algorithm. 
a)

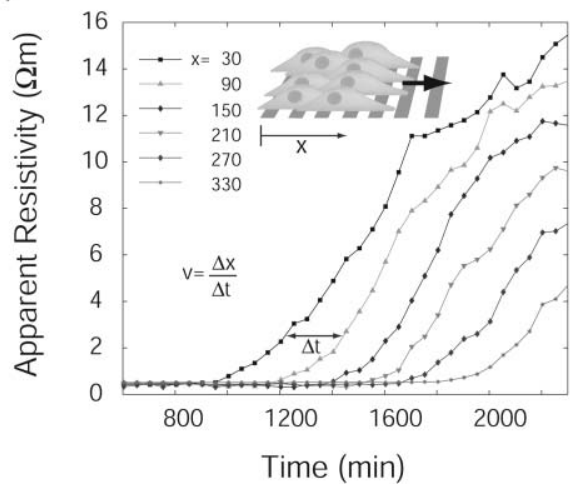

b)

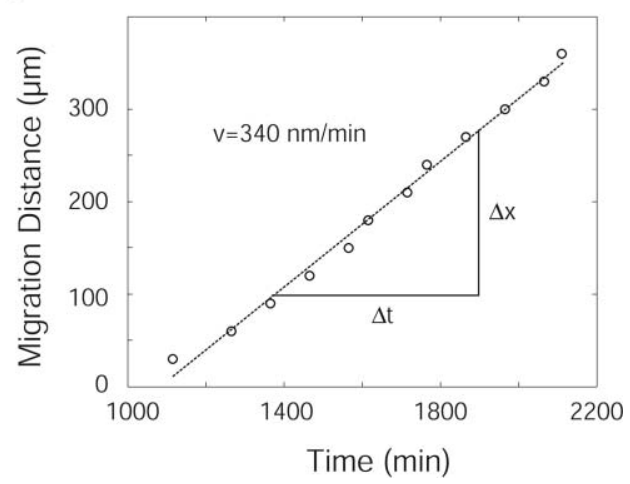

Fig. 7 (a) As the cells moved across the pick-up electrodes the impedance increased. (b) Plotting the midpoint of the electrode configuration versus the time at which the apparent resistivity had reached a certain value, the migration speed could be determined.

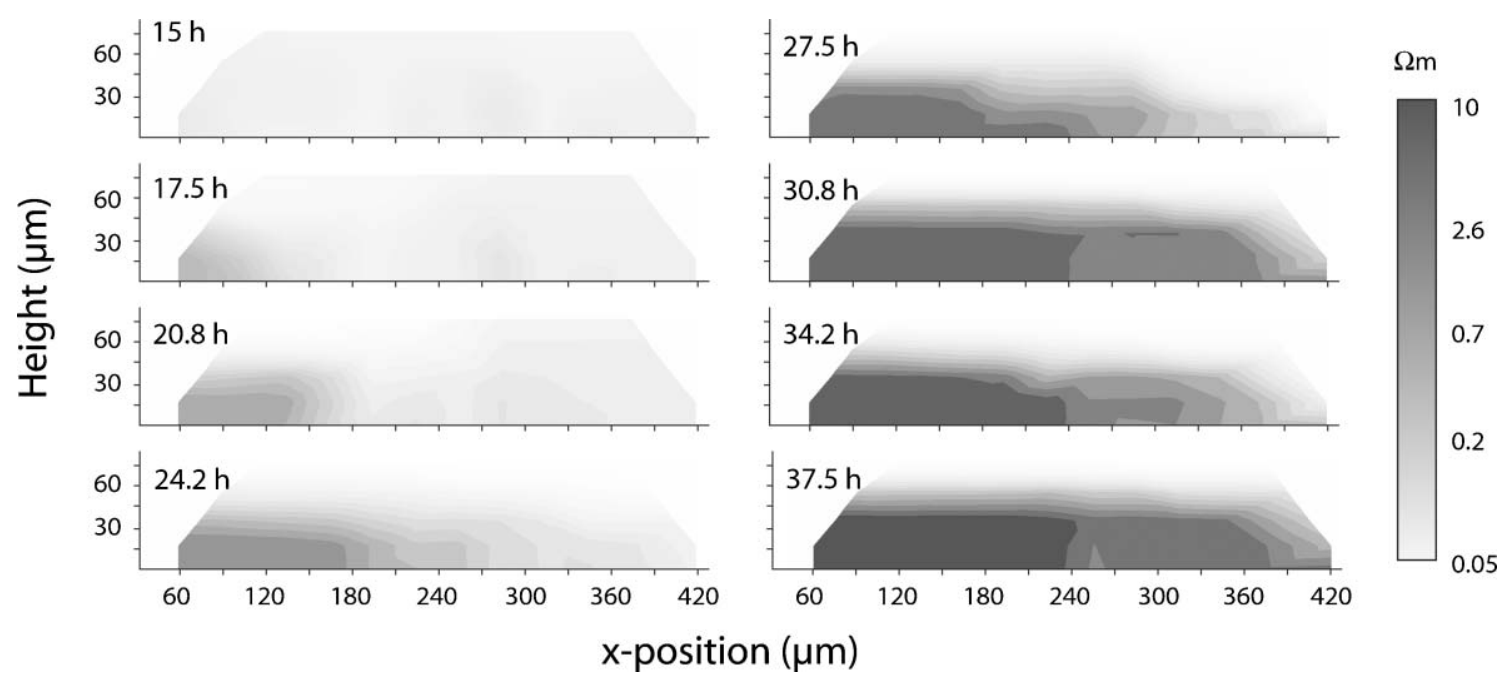

Fig. 8 Time-lapse impedance imaging of cell migration from the left to the right over a time period of 23 hours. The cells are more resistive than the surrounding medium and therefore appear as dark regions in the image. The average RMS error in the inversion was $20.3 \%$.

\section{Summary and outlook}

We developed an imaging device based on an array of rectangular microelectrodes, on which we cultured human epithelial stem cells. Using a software program developed for surveying glaciers and gold mines, we managed to reconstruct the resistivity on top of the electrodes. This allowed us to monitor migration and stratification of the cells in culture, and create $2 \mathrm{D}$ images of migrating cells.

In traditional in vitro wound-healing studies, the migration results are presented as a series of images, and in order to find a more quantitative measure of the migration velocity sophisticated image processing is usually carried out. ${ }^{30} \mathrm{We}$ suggest that these studies could be done more efficiently using electrode arrays, even without image reconstruction. Using an array of microelectrodes, the migration velocities can be studied over a longer period of time compared to singleelectrode techniques such as the ECIS technique, ${ }^{31}$ since the sensing surface is larger. Furthermore, the velocities can be calculated using straightforward regression analysis instead of dealing with obscure time constants.
We believe that the successful miniaturization of the resisitivity imaging technique will open up new areas of research and clinical applications. In the pharmaceutical sciences, it should be possible to pin down the action of pharmaceutical compounds to a specific layer in a threedimensional tissue culture. Such techniques could then replace the traditional TER estimate of a global increase or decrease in resistance. Furthermore, implantable electrode arrays should permit monitoring of cell migration and tissue dynamics, such as carcinogenesis and metastasis or scar tissue formation on neurostimulating electrodes.

At present, only single-frequency measurements are possible if the four-point resistance changes an order of magnitude. Future work should focus on developing electronics that can increase the bandwidth of the four-point measurements, to enable multi-frequency imaging.

Finally, the tomography algorithms and hardware could easily be integrated with existing multielectrode arrays used to record neural spiking. ${ }^{32}$ By simultaneously measuring the resistivity distribution and neural spiking, the 
localization of the firing neurons could be determined more accurately.

\section{References}

1 H. Galler, Pfluegers Arch. Gesamte Physiol. Menschen Tiere, 1912, 149(1/3), 156-174.

2 H. H. Ussing, Physiol. Rev., 1949, 29(2), 127-155.

3 M. S. Balda, J. A. Whitney, C. Flores, S. González, M. Cereijido and K. Matter, J. Cell Biol., 1996, 134(4), 1031.

4 L. Marescot, M. H. Loke, D. Chapellier, R. Delaloye, C. Lambiel and E. Reynard, Near Surf. Geophys., 2003, 1(1), 57-67.

5 A. Binley, G. Cassiani, R. Middleton and P. Winship, J. Hydrol., 2002, 267(3-4), 147-159.

6 K. Spitzer and M. Chouteau, Geophysics, 2003, 68(2), 453-463.

7 P. Metherall, D. C. Barber, R. H. Smallwood and B. H. Brown, Nature, 1996, 380(6574), 509.

8 M. Cheney, D. Isaacson and J. C. Newell, SIAM Rev., 1999, 41(1), 85-101.

9 A. Vonk-Noordegraaf, Physiol. Meas., 2000, 21(2), 285-293.

10 T. Tidswell, A. Gibson, R. H. Bayford and D. S. Holder, Neuroimage, 2001, 13(2), 283-294.

11 B. H. Brown, D. C. Barber and A. D. Seagar, Clin. Phys. Physiol. Meas., 1985, 6(2), 109-121.

12 K. T. C. Chai, P. A. Hammond and D. R. S. Cumming, Sens. Actuators, B, 2005, 111-112, 305-309.

13 T. York, L. Sun, C. Gregory and J. Hatfield, Sens. Actuators, A, 2004, 110(1-3), 213-218.

14 H. Griffiths, M. G. Tucker, J. Sage and W. G. Herrenden-Harker, Physiol. Meas., 1996, 17, A15-A24.
15 D. M. Jones, R. H. Smallwood, D. R. Hose and B. H. Brown, Electron. Lett., 2001, 37(25), 1515.

16 L. Lu, Med. Biol. Eng. Comput., 1996, 34(2), 122.

17 R. V. Davalos, D. M. Otten, L. M. Mir and B. Rubinsky, IEEE Trans. Biomed. Eng., 2004, 51(5), 761-767.

18 I. Giaever and C. R. Keese, Proc. Natl. Acad. Sci. U. S. A., 1991, 88(17), 7896-7900.

19 A. Roy and A. Apparao, Geophysics, 1971, 36(5), 943.

20 L. S. Edwards, Geophysics, 1977, 42(5), 1020.

21 H. P. Schwan and C. D. Ferris, Rev. Sci. Instrum., 1968, 39(4), 481.

22 J. M. Torrents and R. Pallas-Areny, IEEE Trans. Instrum. Meas., 2002, 51(2), 310.

23 C. Littwitz, T. Ragheb and L. A. Geddes, Med. Biol. Eng. Comput., 1990, 28(6), 587-590.

24 P. Linderholm and P. Renaud, Lab Chip, 2005, 5(12), 1416.

25 R. Schinzinger and P. A. A. Laura, Conformal Mapping: Methods and Applications, Elsevier, Amsterdam, 1991.

26 E. Gersing, Biomed. Tech., 1991, 36(1-2), 6.

27 L. R. Lines and S. Treitel, Geophys. Prospect., 1984, 32(2), 159-186.

28 C. Holzapfel, J. Vienken and U. Zimmermann, J. Membr. Biol., 1982, 67(1), 13-26.

29 R. L. Waikel, Y. Kawachi, P. A. Waikel, X.-J. Wang and D. R. Roop, Nat. Genet., 2001, 28(2), 165-168.

30 Z. Környei, A. Czirok, T. Vicsek and E. Madarasz, J. Neurosci. Res., 2000, 61(4), 421-429.

31 J. Wegener, C. R. Keese and I. Giaever, Exp. Cell Res., 2000, 259(1), 158-166.

32 S. I. Morefield, E. W. Keefer, K. D. Chapman and G. W. Gross, Biosens. Bioelectron., 2000, 15(7-8), 383-396. 